\title{
Enhancement of Fibrinolysis In Vitro by Ultrasound
}

\author{
Charles W. Francis, Páll T. Önundarson, Edwin L. Carstensen, * Ales Blinc, Richard S. Meltzer, * \\ Kart Schwarz, * and Victor J. Marder \\ Hematology and ${ }^{*}$ Cardiology Units, Department of Medicine and ${ }^{\ddagger}$ Department of Electrical Engineering and Rochester Center for \\ Biomedical Ultrasound, University of Rochester, Rochester, New York 14642
}

\begin{abstract}
The effect of ultrasound on the rate of fibrinolysis has been investigated using an in vitro system. Plasma or blood clots containing a trace label of ${ }^{125} \mathrm{I}$ fibrin were suspended in plasma containing plasminogen activator and intermittently exposed to continuous wave 1-MHz ultrasound at intensities up to $8 \mathrm{~W} /$ $\mathrm{cm}^{2}$. Plasma clot lysis at $1 \mathrm{~h}$ with $1 \mu \mathrm{g} / \mathrm{ml}$ recombinant tissue plasminogen activator (rt-PA) was $12.8 \pm 1.2 \%$ without ultrasound and was significantly $(P=0.0001)$ increased by exposure to ultrasound with greater lysis at $1 \mathrm{~W} / \mathrm{cm}^{2}(18.0 \pm 1.4 \%)$, $2 \mathrm{~W} / \mathrm{cm}^{2}(19.3 \pm 0.7 \%), 4 \mathrm{~W} / \mathrm{cm}^{2}(22.8 \pm 1.8 \%)$, and $8 \mathrm{~W} / \mathrm{cm}^{2}$ $(58.7 \pm 7.1 \%)$. Significant increases in lysis were also seen with urokinase at ultrasound intensities of $2 \mathrm{~W} / \mathrm{cm}^{2}$ and above. Exposure of clots to ultrasound in the absence of plasminogen activator did not increase lysis. Ultrasound exposure resulted in a marked reduction in the rt-PA concentration required to achieve an equivalent degree of lysis to that seen without ultrasound. For example, 15\% lysis occurred in $1 \mathrm{~h}$ at $1 \mu \mathrm{g} / \mathrm{ml} \mathrm{rt-PA}$ without ultrasound or with $0.2 \mu \mathrm{g} / \mathrm{ml}$ with ultrasound, a fivefold reduction in concentration. Ultrasound at $1 \mathrm{~W} / \mathrm{cm}^{2}$ and above also potentiated lysis of retracted whole blood clots mediated by rt-PA or urokinase. The maximum temperature increase of plasma clots exposed to $4 \mathrm{~W} / \mathrm{cm}^{2}$ ultrasound was only $1.7^{\circ} \mathrm{C}$, which could not explain the enhancement of fibrinolysis. Ultrasound exposure did not cause mechanical fragmentation of the clot into sedimentable fragments, nor did it alter the sizes of plasmic derivatives as demonstrated by SDS polyacrylamide gel electrophoresis. We conclude that ultrasound at 1 MHz potentiates enzymatic fibrinolysis by a nonthermal mechanism at energies that can potentially be applied and tolerated in vivo to accelerate therapeutic fibrinolysis. (J. Clin. Invest. 1992. 90:2063-2068.) Key words: fibrinolytic therapy • fibrinolysis - ultrasound - fibrin - fibrinogen - tissue plasminogen activator $\bullet$ urokinase-like plasminogen activator
\end{abstract}

\section{Introduction}

The fibrinolytic system is carefully regulated physiologically to remodel and remove fibrin deposits, but this process can be accelerated pharmacologically in the treatment of thrombotic diseases by the administration of plasminogen activators. In

Address correspondence to Charles W. Francis, M.D., Hematology Unit, P.O. Box 610, University of Rochester Medical Center, 601 Elmwood Avenue, Rochester, NY 14642.

Received for publication 14 February 1992 and in revised form 3 June 1992.

J. Clin. Invest.

(c) The American Society for Clinical Investigation, Inc.

$0021-9738 / 92 / 11 / 2063 / 06 \$ 2.00$

Volume 90, November 1992, 2063-2068 patients with acute myocardial infarction, fibrinolytic therapy accelerates the dissolution of coronary artery thrombi and reduces mortality and morbidity (1-6). The speed of clot lysis is an important determinant of therapeutic success in the treatment of myocardial infarction $(5,7)$, since tissue necrosis increases with the duration of arterial occlusion and ischemia. Consequently, strategies to improve outcome of fibrinolytic therapy have focused on achieving rapid clot lysis by optimizing dosage regimens of plasminogen activators and also by the adjunctive use of anticoagulants and platelet function inhibitors (8).

As a possible alternative, we have investigated the potential of ultrasound to accelerate fibrinolysis. Ultrasound at low frequencies of $25 \mathrm{KHz}$ has been reported to cause nonenzymatic clot disruption in vitro (9-11) and to accelerate vascular reperfusion when applied via vascular catheters to thrombi in dogs (10-13). The noninvasive percutaneous application of ultrasound at $250 \mathrm{KHz}$ may also accelerate recombinant tissue plasminogen activator (rt-PA) ${ }^{1}$-mediated lysis of experimentally induced arterial thrombi in dogs (14). We have developed an in vitro system to investigate the effects of ultrasound on fibrinolysis and have characterized the influence of $1-\mathrm{MHz}$ ultrasound at varying intensities on blood clot lysis mediated by rt-PA or two-chain urokinase-like plasminogen activator (UK).

\section{Methods}

Clot preparation. Fresh frozen plasma anticoagulated with acid citrate dextrose was obtained from five donors through the American Red Cross (Rochester Region, Rochester, NY), pooled, aliquoted and stored at $-70^{\circ} \mathrm{C}$ until use. Blood was obtained by antecubital venipuncture from normal volunteers and collected into a plastic syringe without anticoagulant and used immediately to prepare clots. Human fibrinogen (grade L; Helena Laboratories, Beaumont, TX) was ${ }^{125} \mathrm{I}$-radiolabeled using the iodogen technique (15) to a specific activity of 50 $\mu \mathrm{Ci} / \mathrm{mg}$, and unbound isotope was separated by gel filtration. The labeled fibrinogen was $>90 \%$ clottable. Radiolabeled retracted whole blood clots were prepared by adding $160 \mu \mathrm{l}$ of blood and a trace amount of ${ }^{125} \mathrm{I}$-labeled fibrinogen to glass tubes and incubating at $37^{\circ} \mathrm{C}$ for $2 \mathrm{~h}$. The retracted clots were then removed from the serum, washed once with $0.1 \mathrm{M}$ sodium chloride, $0.05 \mathrm{M}$ Tris buffer, $\mathrm{pH} 7.4$, containing 5 $\mathrm{mM}$ glucose and transferred to nitrocellulose tubes for fibrinolysis experiments. Plasma clots were formed by adding $160 \mu \mathrm{l}$ of plasma and a trace amount of ${ }^{125} \mathrm{I}$-labeled fibrinogen to nitrocellulose tubes and clotting by the addition of $50 \mathrm{mM}$ calcium chloride and $10 \mathrm{U} / \mathrm{ml}$ bovine thrombin (Calbiochem Corp., La Jolla, CA).

Ultrasound apparatus. Clot lysis experiments were performed in thin-walled 8-mm diameter nitrocellulose tubes (Beckman Instruments, Inc., Palo Alto, CA). The attenuation of the wall was $<0.8 \mathrm{db}$ at $1 \mathrm{MHz}$ as determined by insertion loss measurements when the water-filled tube was placed between the source and a needle hydro-

1. Abbreviations used in this paper: rt-PA, recombinant tissue plasminogen activator; UK, two-chain urokinase-like plasminogen activator. 

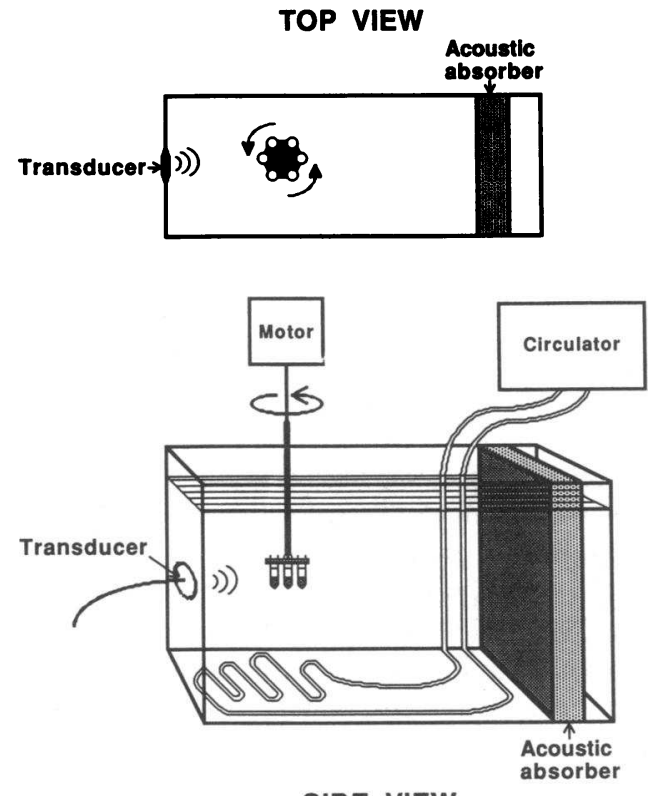

SIDE VIEW

Figure 1. Experimental apparatus. A plexiglass tank was fitted with a transducer and filled with water. An acoustic absorber was placed at the opposite end of the tank from the transducer, and water was circulated to maintain a constant temperature. Up to six test tubes containing clot and plasma were placed in a circular rack and rotated through the site of maximum intensity of the ultrasound field.

phone (type 80-0.5-4.0; Imotek GmbH, Würselen, Germany). The tubes were sealed and placed in a round test tube rack with a diameter of $2.5 \mathrm{~cm}$ containing a maximum of six tubes (Fig. 1). The rack was immersed in a tank of water maintained at $37^{\circ} \mathrm{C}$, and the tubes were positioned at the location of maximum ultrasound intensity. The test tube rack was rotated at 6-8 rpm during fibrinolysis experiments to give equivalent exposure to all tubes. The source of ultrasound was a 1-in. diameter, 1-MHz, piezoelectric transducer driven in the continuous wave mode. Samples were located at a distance of $8 \mathrm{~cm}$ from the source, at which position the $-6 \mathrm{db}$ beamwidth of the sound field was $6.5 \mathrm{~mm}$. The sound fields were measured with a bilaminar, polyvinyldifluoride membrane hydrophone (Marconi Research Center, Chelmsford, UK) on a weekly or biweekly basis and monitored continuously during exposures through the radio frequency driving voltage on the crystal. A 3-cm thick block of rubber was used as an acoustic absorber to limit ultrasound reflection.

Clot lysis experiments. Retracted blood clots were transferred to the nitrocellulose exposure tubes after formation, but plasma clots were formed directly within the exposure tubes. Thus, the blood clots were free to move in the tube and were surrounded by plasma, whereas the plasma clots were fixed and directly exposed to plasma only on the upper surface. To prevent clots from occupying the bottom of the tube where refraction would make definition of the sound field more difficult, the curved part of the tube was filled with $150 \mu l$ of polymerized $3 \%$ agarose. Citrated plasma $(160 \mu \mathrm{l})$ containing $2 \mathrm{U} / \mathrm{ml}$ heparin (Riker Labs, Inc., Northridge, CA) was added, and the tubes were placed in the sample holder within the ultrasound apparatus. UK (Abbott Laboratories, No. Chicago, IL) or rt-PA (Genentech, So. San Francisco, CA ) was added to the plasma, and the clots were incubated in the rotating apparatus. At desired times, tubes were removed from the apparatus, fibrinolysis was stopped by adding $500 \mathrm{U} / \mathrm{ml}$ aprotinin (Mobay Chemical Co., New York, NY) to inhibit plasmin, and samples were mixed and centrifuged at $2300 \mathrm{~g}$ for $5 \mathrm{~min}$. An aliquot of the supernatant was removed, and the percent lysis of the clot was calculated from the solubilization of radiolabel. For some experiments, plasminogen activator was added to the plasma prior to clotting so that it was incorporated throughout the clot. For these experiments no plasminogen activator was added to the suspending plasma.

Electrophoresis. SDS polyacrylamide gel electrophoresis was performed using 4-10\% to gradient gels and a discontinuous buffer system as described (16).

Statistical analysis. The statistical significance of differences between means was tested using a two-tailed Student's $t$ test for unpaired data. One-way analysis of variance (ANOVA) was used for comparisons involving multiple groups and the Dunnett $t$ test for post-hoc comparison between groups.

\section{Results}

Plasma clots were overlaid with plasma containing $1 \mu \mathrm{g} / \mathrm{ml}$ rt-PA, and the extent of clot lysis was measured at $1 \mathrm{~h}$ with or without exposure to 1-MHz ultrasound (Fig. 2). Clot lysis with rt-PA was $12.8 \pm 1.2 \%$ without ultrasound and was increased with ultrasound to $18.0 \pm 1.4 \%$ at $1 \mathrm{~W} / \mathrm{cm}^{2}, 19.3 \pm 0.7 \%$ at 2 $\mathrm{W} / \mathrm{cm}^{2}, 22.8 \pm 1.8 \%$ at $4 \mathrm{~W} / \mathrm{cm}^{2}$, and to $58.7 \pm 7.1 \%$ at $8 \mathrm{~W} /$ $\mathrm{cm}^{2}$. One-way ANOVA indicated a significant $(P=0.0001)$ difference between means with increases at $4 \mathrm{~W} / \mathrm{cm}^{2}(P$ $<0.05)$ and $8 \mathrm{~W} / \mathrm{cm}^{2}(P<0.01)$. Significant increases in lysis were also seen with $\mathrm{UK}(250 \mathrm{U} / \mathrm{ml})$ at ultrasound intensities of $2 \mathrm{~W} / \mathrm{cm}^{2}$ and above. In the absence of activator, there was lysis of $3 \%$ or less at $1 \mathrm{~h}$ with any ultrasound intensity.

The ultrasonic potentiation of fibrinolysis was present by $15 \mathrm{~min}$ of exposure (Fig. 3), and the increase persisted at 30 min, $1 \mathrm{~h}$, and $2 \mathrm{~h}$. At $4 \mathrm{~h}$ there was nearly complete clot lysis with or without ultrasound. Exposure to ultrasound resulted in marked shortening of the time to achieve equivalent degrees of clot lysis. For example, in the absence of ultrasound, $75 \mathrm{~min}$ was required to lyse $20 \%$ of the clot, and this was reduced to less

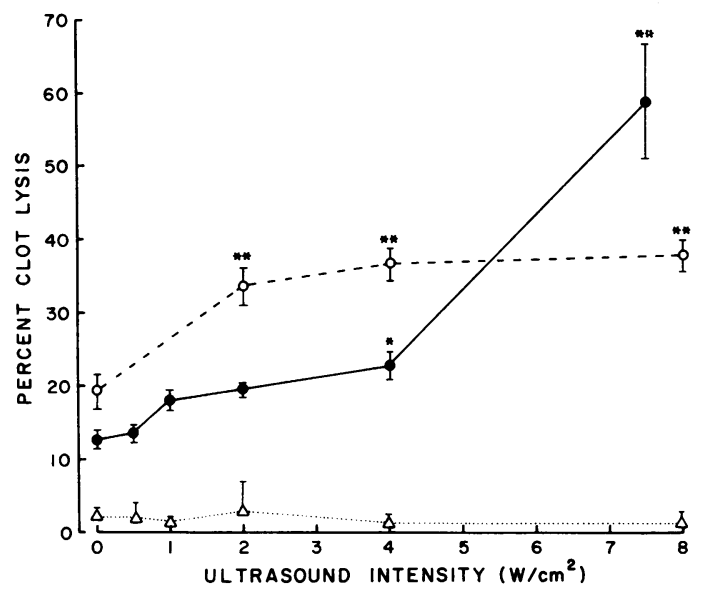

Figure 2. Effect of ultrasound on lysis of plasma clots. Plasma clots were formed in nitrocellulose test tubes from citrated plasma containing ${ }^{125} \mathrm{I}$-fibrinogen by the addition of $50 \mathrm{mM}$ calcium chloride and $10 \mathrm{U} / \mathrm{ml}$ thrombin. The clots were overlaid with heparinized plasma containing $1 \mu \mathrm{g} / \mathrm{ml} \mathrm{rt-PA} \mathrm{(solid} \mathrm{line)} \mathrm{or} 250 \mathrm{U} / \mathrm{ml} \mathrm{UK}$ (dashed line), and then incubated at $37^{\circ} \mathrm{C}$ for $1 \mathrm{~h}$ without ultrasound or with 1 $\mathrm{MHz}$ ultrasound at intensities up to $8 \mathrm{~W} / \mathrm{cm}^{2}$. In the absence of plasminogen activator, there was less than $5 \%$ release of radiolabel at $1 \mathrm{~h}$ at all ultrasound intensities. Each point represents the mean \pm SEM of lysis of six or more experiments. One-way ANOVA indicated that the differences between means was significant at $P=0.0001$. Statistical significance in comparison with no ultrasound exposure is indicated by ${ }^{*} P<0.05 ;{ }^{* *} P<0.01$; using the Dunnett $t$ test. Dotted line shows lysis with ultrasound and no activator. 


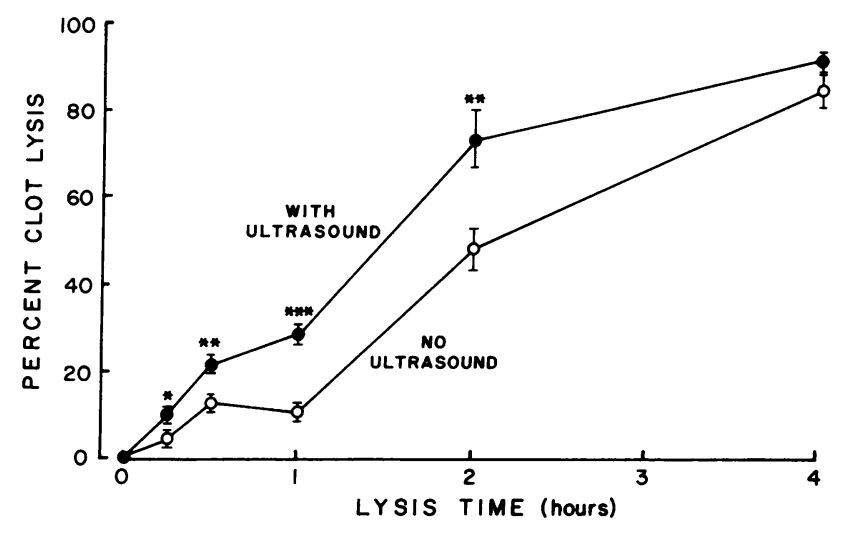

Figure 3. Time course of clot lysis in the presence or absence of ultrasound. Radiolabeled plasma clots were formed in nitrocellulose tubes and overlaid with heparinized plasma containing $1 \mu \mathrm{g} / \mathrm{ml} \mathrm{rt}-\mathrm{PA}$ and incubated at $37^{\circ} \mathrm{C}$ in the presence of $4 \mathrm{~W} / \mathrm{cm}^{2}$ ultrasound or without ultrasound. Each point represents the mean \pm SEM of six experiments. Statistical significance is indicated by: ${ }^{*} P<0.03$; ${ }^{* *} P$ $\left.<0.01 ;{ }^{* *} P<0.001\right)$.

than $30 \mathrm{~min}$ by exposure to $4 \mathrm{~W} / \mathrm{cm}^{2}$ ultrasound. Clot lysis of $70 \%$ was reached by 210 min without ultrasound, compared to $\sim 115$ min with ultrasound.

Ultrasound potentiated clot lysis over a range of rt-PA concentrations up to $1 \mu \mathrm{g} / \mathrm{ml}$ (Fig. 4). Significant increases in clot lysis at $1 \mathrm{~h}$ were seen at rt-PA concentrations of $0.25,0.5$, and 1 $\mu \mathrm{g} / \mathrm{ml}$ with exposure to $4 \mathrm{~W} / \mathrm{cm}^{2}$ ultrasound $(P<0.0005)$. This resulted in a marked reduction in the rt-PA concentration required to achieve equivalent degrees of lysis. For example, $15 \%$ lysis occurred in $1 \mathrm{~h}$ at an rt-PA concentration of $1 \mu \mathrm{g} / \mathrm{ml}$ in the absence of ultrasound, but this degree of lysis occurred at $\sim 0.2 \mu \mathrm{g} / \mathrm{ml}$ in the presence of ultrasound, a fivefold reduction in rt-PA concentration.

Ultrasound also potentiated rt-PA mediated lysis of retracted whole blood clots, with significant increases in 1-h lysis

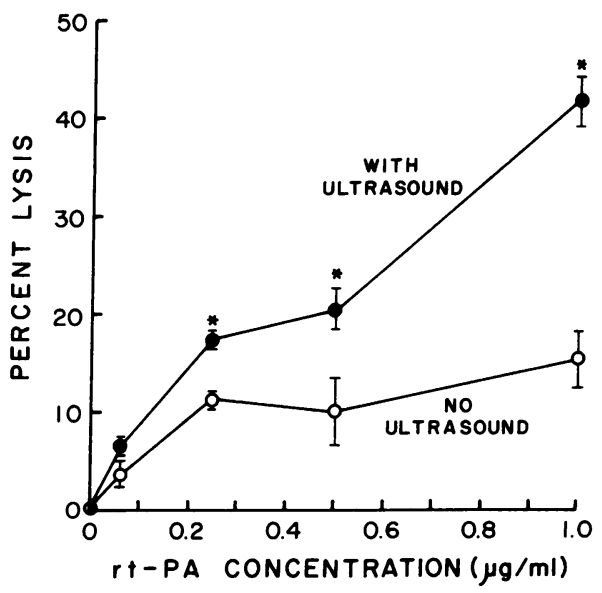

Figure 4. Effect of ultrasound on clot lysis with varying concentrations of rt-PA. Radiolabeled plasma clots were incubated for $1 \mathrm{~h}$ at $37^{\circ} \mathrm{C}$ in normal citrated plasma containing rt-PA at concentrations up to $1 \mu \mathrm{g} / \mathrm{ml}$. Clots were exposed to ultrasound at $4 \mathrm{~W} / \mathrm{cm}^{2}$ or received no ultrasound. Individual points represent the mean \pm SEM of six experiments. Lysis was significantly greater in the presence of ultrasound at $0.25 \mu \mathrm{g} / \mathrm{ml}, 0.5 \mu \mathrm{g} / \mathrm{ml}$, and $1 \mu \mathrm{g} / \mathrm{ml}(P<0.0005$ for each $)$.

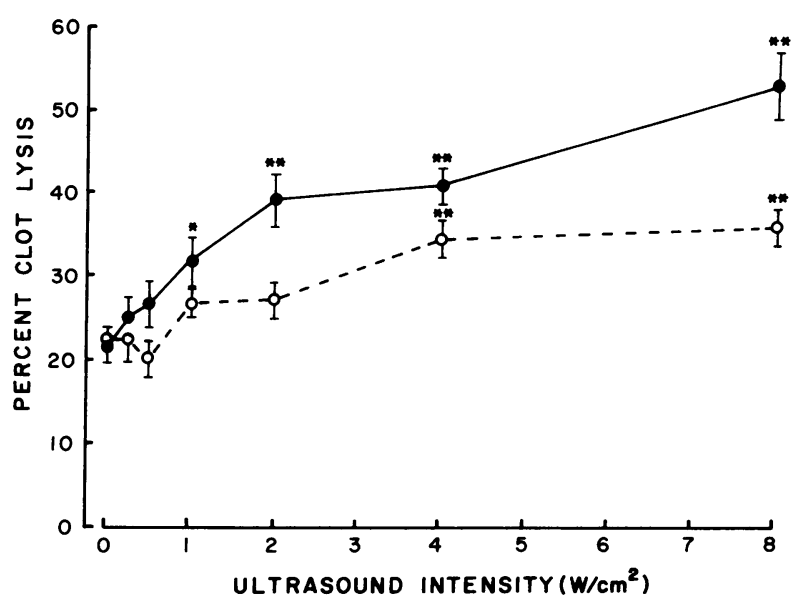

Figure 5. Effect of ultrasound on lysis of whole blood clots. Radiolabeled retracted whole blood clots were suspended in plasma containing $1 \mu \mathrm{g} / \mathrm{ml} \mathrm{rt-PA} \mathrm{(solid} \mathrm{lines)} \mathrm{or} 250 \mathrm{U} / \mathrm{ml} \mathrm{UK}$ (dashed line) and incubated at $37^{\circ} \mathrm{C}$ for $1 \mathrm{~h}$ in the presence of no ultrasound, or ultrasound at intensities of up to $8 \mathrm{~W} / \mathrm{cm}^{2}$. The points represent the mean \pm SEM of 9-12 experiments. One-way ANOVA indicated that the differences between means were significant $(P=0.0001)$. Statistical significance in comparison with no ultrasound is indicated as: ${ }^{*} P<0.05 ;{ }^{*} P<0.01$ using the Dunnett $t$ test.

at ultrasound intensities of $1 \mathrm{~W} / \mathrm{cm}^{2}$ and above (Fig. 5). Clot lysis increased from $21.1 \pm 1.6 \%$ in the absence of ultrasound to $39.0 \pm 3.3 \%$ at $4 \mathrm{~W} / \mathrm{cm}^{2}$ and $52.8 \pm 4.1$ at $8 \mathrm{~W} / \mathrm{cm}^{2}$. Clot lysis was also potentiated using UK $(250 \mathrm{U} / \mathrm{ml})$ with an increase from $22.2 \pm 1.6 \%$ at $1 \mathrm{~h}$ in the absence of ultrasound to $35.8 \pm 2.1 \%$ at $8 \mathrm{~W} / \mathrm{cm}^{2}$.

Exposure of plasma clots to continuous ultrasound resulted in heating (Fig. 6). These data were obtained with thermocouples embedded in clots rotating through the ultrasound beam. The temperature rose to a plateau in approximately $2 \mathrm{~min}$ when the rates of heating and heat dissipation became approximately equal. The temperature increase was $1^{\circ} \mathrm{C}$ at $2 \mathrm{~W} / \mathrm{cm}^{2}$, $1.7^{\circ} \mathrm{C}$ at $4 \mathrm{~W} / \mathrm{cm}^{2}$, and $3^{\circ} \mathrm{C}$ at $8 \mathrm{~W} / \mathrm{cm}^{2}$.

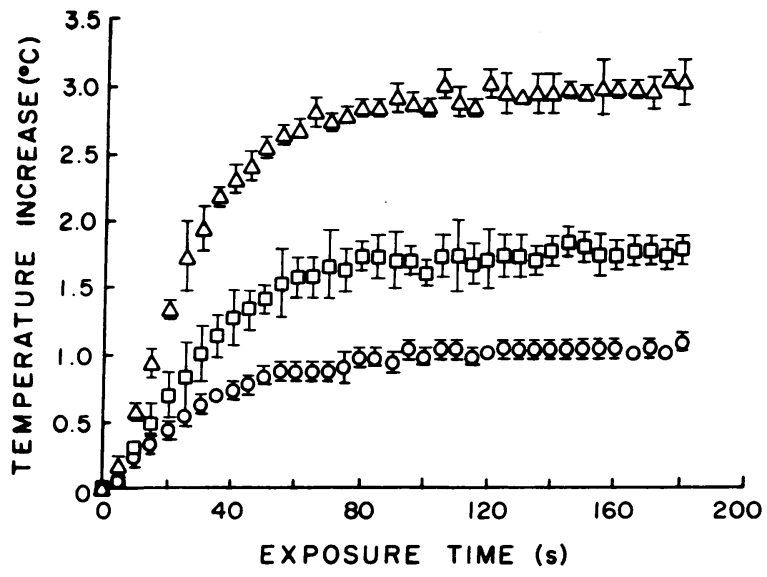

Figure 6. Heating of plasma clots in nitrocellulose tubes rotating through the ultrasound beam. Clots were formed around a thermocouple and insonified with continuous wave ultrasound at 2,4 , and $8 \mathrm{~W} / \mathrm{cm}^{2}$. The data represent the mean \pm SEM of three experiments at each intensity. 
In separate experiments the temperature dependence of fibrinolysis with rt-PA was determined. The percentage lysis at 1 $\mathrm{h}$ with $1 \mu \mathrm{g} / \mathrm{ml} \mathrm{rt}-\mathrm{PA}$ increased linearly with temperature from $13.2 \pm 5.6 \%$ at $24^{\circ} \mathrm{C}$ to a maximum of $40.1 \pm 5.8 \%$ at $50^{\circ} \mathrm{C}$. In this temperature range a linear correlation coefficient of 0.89 was found between temperature and percent lysis. Linear regression analysis derived a slope corresponding to a $1.1 \%$ increase in lysis per $1^{\circ} \mathrm{C}$. At temperatures higher than $50^{\circ} \mathrm{C}$ the lysis rate decreased rapidly and was only $29.2 \pm 5.4 \%$ at $55^{\circ} \mathrm{C}$.

Since ultrasound induces oscillations of hydrostatic pressure, we examined the effects of increased pressure on fibrinolysis. No significant difference in plasma clot lysis was observed with $1 \mu \mathrm{g} / \mathrm{ml} \mathrm{rt}-\mathrm{PA}$ at $37^{\circ} \mathrm{C}$ at 1 atm pressure compared to 4.3 atm, which is similar to the acoustic pressure amplitude in a sound field at $8 \mathrm{~W} / \mathrm{cm}^{2}$.

To determine if the clot was disrupted into macroscopic fragments by ultrasound, the lysate was centrifuged at $15,000 \mathrm{~g}$ for $2 \mathrm{~min}$ to sediment particulate material. All radioactivity remained in solution, indicating that large fragments were not present. Lysates obtained in the presence or absence of ultrasound were also examined by SDS $4 / 10 \%$ polyacrylamide gel electrophoresis. The sizes and distribution of fragments were the same, indicating that ultrasound exposure did not alter the pattern of plasmic degradation of the fibrin substrate. Addition of $10 \%$ trichloroacetic acid to the supernatant containing degradation products precipitated $95 \%$ of radioactivity, indicating that ultrasound did not release protein-bound iodine.

We considered that ultrasonic potentiation of fibrinolysis could be due to increased transport of activator into the clot from the surrounding plasma, thereby accelerating enzymatic degradation. To examine this possibility, activator was added to plasma which was then immediately clotted. Fibrinolysis was potentiated by ultrasound to a similar extent (Fig. 7) as in experiments with activator only in the surrounding plasma, indicating that enhanced transport of enzyme from solution

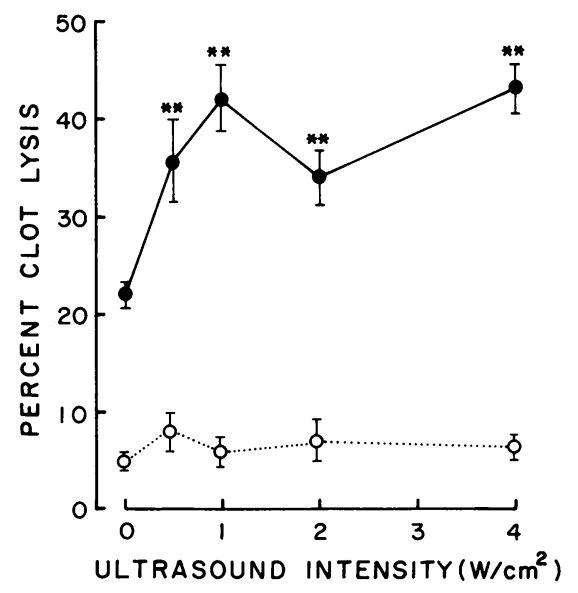

Figure 7. Effect of ultrasound on lysis of clots containing rt-PA incorporated throughout. Solid line: Citrated plasma containing ${ }^{125} \mathrm{I}-\mathrm{fi}-$ brinogen and $75 \mathrm{ng} / \mathrm{ml}$ of rt-PA was clotted by addition of $10 \mathrm{U} / \mathrm{ml}$ thrombin and $50 \mathrm{mM}$ calcium chloride. The clot was incubated at $37^{\circ} \mathrm{C}$ without ultrasound or with exposure to ultrasound at intensities up to $4 \mathrm{~W} / \mathrm{cm}^{2}$. The points represent the mean \pm SEM of 3-15 experiments. One-way ANOVA indicated that the difference between means was significant at $P=0.0001$. Statistical significance in comparison with no ultrasound is indicated by: ${ }^{* *} P<0.01$ as calculated using the Dunnett $t$ test. Dashed line: Ultrasound with no activator. into the clot did not completely explain the increased rate of fibrinolysis with ultrasound.

\section{Discussion}

The results presented in this report demonstrate that the exposure of clots to $1-\mathrm{MHz}$ ultrasound in vitro accelerates enzymatic fibrinolysis induced by rt-PA or UK. The experimental system (Fig. 1) generates continuous wave ultrasound but uses a rotating sample holder, so that individual clots receive intermittent ultrasound exposure. The experimental design insures accurate determination of clot lysis rate by solubilization of radiolabeled fibrin, and allows measurement of ultrasound field strength at the site of the dissolving clot. Significant increases in lysis rates of both plasma and whole blood clots were observed at $1 \mathrm{~W} / \mathrm{cm}^{2}$, and greater acceleration occurred at higher intensities of up to $8 \mathrm{~W} / \mathrm{cm}^{2}$. Ultrasonic augmentation of fibrinolysis was seen as early as $15 \mathrm{~min}$ (Fig. 3 ) and resulted in a marked reduction in the concentration of activator required to achieve lysis (Fig. 4).

The effects of ultrasound described in this report differ from those in which lower frequency, high intensity ultrasound has been used to mechanically disrupt clots. Ultrasound at 20 $\mathrm{kHz}$ and energies of up to $20 \mathrm{~W}$ delivered by a thin wire can mechanically disrupt clots (9-11) or atherosclerotic plaque $(17,18)$ in vitro, producing fragments with diameters varying from 2.5 to $800 \mu \mathrm{m}$. This approach has been applied to dog models of femoral artery or femoral vein thrombosis in vivo using a catheter to guide the ultrasonic wire to the intravascular thrombus (10-13). Activation of the wire can disrupt the thrombus within minutes into particles which can either be aspirated or embolized distally. Percutaneous, transvascular ultrasonic disruption of a femoral artery thrombus has been reported in a single patient (13), and ultrasound has also been used to recanalize arteries obstructed by atherosclerosis (19, 20). In general, few complications have been reported in dog models, although heating of the catheter may occur (13). Also, perforation of the vessel by the wire has been reported (10), and histologic evidence of intimal disruption and medial dissection has occurred in some cases $(11,12)$. The latter changes may be related to the catheterization and other procedures needed to produce the clot and be independent of the ultrasound exposure. Using an approach similar to ours, Kudo (14) has reported ultrasonic enhancement of rt-PA mediated fibrinolysis in a dog model. Thrombi were formed in femoral arteries of dogs, and there was significant shortening of the time before rt-PA-mediated recanalization in limbs that received noninvasive percutaneous application of $200-\mathrm{KHz}$ ultrasound directed to the thrombus. Parameters related to ultrasound exposure or to fibrinolytic agents affecting lysis of thrombus were not investigated in this study, nor was a mechanism proposed to explain the effect.

Ultrasound can cause biologic effects through several mechanisms (21) which should be considered in interpretation of the findings in this report. As fibrinolysis is temperature dependent, heating could accelerate clot lysis. However, ultrasound exposure was associated with only a minimal increase in clot temperature at $4 \mathrm{~W} / \mathrm{cm}^{2}$, insufficient to explain the observed increase in lysis. At $8 \mathrm{~W} / \mathrm{cm}^{2}$ the amplitude of the pressure oscillations in the sound field is 4.8 atm, but fibrinolysis was not accelerated at a hydrostatic pressure of $4.3 \mathrm{~atm}$. This is 
consistent with previous reports that much higher pressure increases are needed to alter most enzymatic reactions (22).

Since heating and static pressure effects cannot explain the enhanced fibrinolysis, nonthermal mechanisms such as acoustic cavitation should be considered. Acoustic cavitation is the expansion and sometimes violent collapse of bubbles resulting from the pressure changes in the medium associated with the passage of the sound wave (23). The thresholds that we have observed for enhanced fibrinolysis are approximately the level that, in water, are associated with the transition of bubble activity from a relatively stable oscillation to a large expansion and violent collapse $(24,25)$, termed "transient cavitation." Above that threshold the temperatures generated in the gas during adiabatic collapse can become great enough to produce free radicals, and shock waves created by the rebound of the bubble can be orders of magnitude greater than the acoustic pressures responsible for the cavitation. Bubbles that produce the most dramatic effects of cavitation with 1-MHz sound fields have a diameter of several microns, which is similar to the pore size in plasma clots $(26,27)$.

Fluid motion caused by cavitation could accelerate fibrinolysis by increasing transport of reactants. Blinc et al. (28) have shown that fibrinolysis in vitro is slow if transport of activator into clot occurs by diffusion, but is more rapid if transport is increased by pressure-induced permeation. This is also consistent with data from animal models (29) and with clinical observations (30) that fibrinolytic therapy is more rapid and successful if activator can be injected into a thrombus rather than if it is infused into the blood proximal to the obstruction. Increased transport of activator from plasma into the clot may have contributed to the accelerated lysis observed in the experiments reported, except for that in Fig. 7 in which activator was present throughout the clot at the time of formation. In that experiment, a lower concentration of rt-PA was required, compared to experiments in which activator was initially present in the surrounding plasma, confirming the importance of activator distribution on lysis rate. However, even with activator distributed through the clot, the accelerated fibrinolysis caused by ultrasound may have been related to transport. This could occur by increasing movement of rt-PA from solution to binding sites on fibrin, a process that was diffusion-limited in the absence of ultrasound.

Potential deleterious biologic effects of ultrasound need to be considered in the possible therapeutic application of these results. For example, exposure of tissues to continuous-wave ultrasound at $8 \mathrm{~W} / \mathrm{cm}^{2}$ or even $4 \mathrm{~W} / \mathrm{cm}^{2}$ would produce unacceptable heating (31). However, the lower temporal average intensities that are effective in vitro are well below the levels commonly used in ultrasonic diathermy. Furthermore, there are no clearly established nonthermal deleterious effects of 1$\mathrm{MHz}$ ultrasound in tissues at temporal peak intensities of 8 $\mathrm{W} / \mathrm{cm}^{2}$ if the temporal average intensities are low enough to prevent significant heating ( 32 ). In addition to potential heating of tissues, ultrasound has been reported to activate platelets in vitro (33-35), but clinical complications resulting from this effect have not been reported. At high intensity and low frequency, ultrasound can damage endothelial cells and cause thrombosis (36-38), but this has not been observed at $1 \mathrm{MHz}$ in patients. Not all effects of ultrasound on vascular function are deleterious, however. Ultrasound has been reported to enhance arterial relaxation (39), an effect that could contribute to reperfusion.
Ultrasound could potentially contribute to therapeutic fibrinolysis by accelerating clot dissolution, decreasing the activator concentration required, or decreasing resistance to fibrinolysis. The speed of the clot dissolution during treatment of arterial thrombosis is a critical determinant of the success of fibrinolytic therapy because longer periods of tissue ischemia result in progressive organ dysfunction and cell death. This is especially evident in treatment of acute myocardial infarction, in which the success of therapy is strongly dependent on the interval between the onset of symptoms and successful myocardial reperfusion $(5,7)$, and interventions that accelerate fibrinolysis could improve therapeutic outcome. Also, bleeding complications during fibrinolytic therapy have been related to both rtPA dose (40) and plasma concentration (41). Since ultrasound reduced the rt-PA concentration required to achieve fibrinolysis in vitro, such an activator-sparing effect could reduce complications of rt-PA therapy. Ultrasound application may also permit clot-specific enhancement of fibrinolysis, since the effect is localized to the volume within the ultrasound beam. The potential benefits of ultrasonic enhancement of fibrinolytic therapy will require further studies to define optimai acoustic exposures and to develop appropriate therapeutic devices for controlled clinical trials in patients with thrombotic disorders.

\section{Acknowledgments}

The authors are indebted to Janet Trudnowski for assistance with these experiments, and to Sally Child and Carol Hartman, Department of Electrical Engineering, University of Rochester, for the ultrasound calibrations and temperature measurements, to Xucai Chen, Ph.D., for engineering assistance, and to Carol Weed for help in preparation of the manuscript.

This work was supported in part by grant HL-30616 from the National Heart, Lung, and Blood Institute, and US Public Health Service grants DK39796 and CA39230, National Institutes of Health, Bethesda, MD.

\section{References}

1. Stampfer, J. J., S. Z. Goldhaber, S. Yusuf, R. Peto, and C. H. Hennekens. 1982. N. Engl. J. Med. 307:1180-1182.

2. Yusuf, S., R. Collins, R. Peto, C. Furberg, M. J. Stampfer, S. Z. Goldhaber, and C. H. Hennekens. 1985. Intravenous and intracoronary fibrinolytic therapy in acute myocardial infarction: overview of results on mortality, reinfarction and side effects from 33 randomized trials. Eur. Heart J. 6:556-585.

3. Gruppo Italiano per lo Studio della Streptochinasi nell'Infarto Miocardico (GISSI). 1987. Long-term effects of intravenous thrombolysis in acute myocardial infarction: final report of the GISSI study. Lancet. ii:871-874.

4. ISIS-2 (Second International Study of Infarct Survival) Collaborative Group. 1988. Randomised trial of intravenous streptokinase, oral aspirin, both, or neither among 17,187 cases of suspected acute myocardial infarction: ISIS-2. Lancet. ii:349-360.

5. Marder, V. J., and S. Sherry. 1988. Thrombolytic therapy: Current status. N. Engl. J. Med. 318:1512-1519 and 1585-1594.

6. AIMS Trial Study Group. 1990. Long-term effects of intravenous anistreplase in acute myocardial infarction: final report of the AIMS study. Lancet. 335:427-431.

7. Chesebro, J. H., G. Knatterud, R. Roberts, J. Borer, L. S. Cohen, J. Dalen, H. T. Dodge, C. F. Francis, D. Hillis, P. Ludbrook, et al. 1987. Thrombolysis in myocardial infarction (TIMI) Trial. Phase I: A comparison between intravenous tissue plasminogen activator and intravenous streptokinase. Clinical findings through hospital discharge. Circulation. 76:142-154.

8. Popma, J. J., and E. J. Topol. 1991. Adjuncts to thrombolysis for myocardial reperfusion. Ann. Intern. Med. 115:34-44.

9. Hong, A. S., J.-S. Chae, S. B. Dubin, S. Lee, M. C. Fishbein, and R. J. Siegel. 1990. Ultrasonic clot disruption: An in vitro study. Am. Heart J. 120:418-422.

10. Rosenschein, U., J. J. Bernstein, E. DiSegni, E. Kaplinsky, J. Bernheim, and L. A. Rozenzajn. 1990. Experimental ultrasonic angioplasty: Disruption of 
atherosclerotic plaque and thrombi in vitro and arterial recanalization in vivo. $J$. Am. Coll. Cardiol. 15:711-717.

11. Ariani, M., M. C. Fishbein, J. S. Chae, H. Sadeghi, T. A. DonMichael, S. B. Dubin, and R. J. Siegel. 1988. Dissolution of peripheral arterial thrombi by ultrasound. Circulation. 84:1680-1688.

12. Trübestein, G., C. Engel, F. Etzel, A. Sobbe, H. Cremer, and U. Stumpff. 1976. Thrombolysis by ultrasound. Clin. Sci. Mol. Med. 51:697s-698s. 544.

13. Stumpff, U. 1979. Ultrasonic thrombolysis. J. Acoust. Soc. Am. 65:541-

14. Kudo, S. 1989. Thrombolysis with ultrasound effect. Tokyo Jikeikai Med. J. 104:1005-1012.

15. Fraker, P. J., and J. R. Speck, Jr. 1978. Protein and cell membrane iodinations with a sparingly soluble chloroamide, 1,3,4,6-tetrachloro-3a,6a-diphenylglycoluril. Biochem. Biophys. Res. Commun. 80:849-857.

16. Francis, C. W., V. J. Marder, and S. E. Martin. 1980. Plasmic degradation of crosslinked fibrin. I. Structural analysis of the particulate clot and identification of new macromolecular-soluble complexes. Blood. 56:456-464.

17. Siegel, R. J., M. C. Fishbein, J. Forrester, K. Moore, E. DeCastro, L. Daykhovsky, and T. A. DonMichael. 1988. Ultrasonic plaque ablation. A new method for recanalization of partially or totally occluded arteries. Circulation. 78:1443-1448.

18. Ernst, A., E. A. Schenk, S. M. Gracewski, T. J. Woodlock, F. G. Murant, H. Alliger, and R. S. Meltzer. 1991. Ability of high-intensity ultrasound to ablate human atherosclerotic plaques and minimize debris size. Am. J. Cardiol. 68:242246.

19. Siegel, R. J., R. K. Myler, D. C. Cumberland, and T. A. DonMichael. 1989. Percutaneous ultrasonic angioplasty: Initial clinical experience. Lancet. ii:772-774.

20. Rosenschein, U., L. A. Rozenszajn, L. Kraus, C. C. Marboe, J. F. Watkins, E. A. Rose, D. David, P. J. Cannon, and J. S. Weinstein. 1991. Ultrasonic angioplasty in totally occluded peripheral arteries. Initial clinical, histological, and angiographic results. Circulation. 83:1976-1986.

21. Porder, J. B., K. N. Porder, and R. S. Meltzer. 1987. Ultrasound bioeffects. Echocardiography. 4:89-99.

22. Laidler, K. J., and P. S. Bunting. 1973. The Chemical Kinetics of Enzyme Action. Clarendon Press, Oxford, pp. 196-232.

23. Flynn, H. G. 1964. Physics of acoustic cavitation in liquids. W. Mason, editor. Physical Acoustics, Vol 1, Part B, Chapter 9. Arademic Press, New York.

24. Flynn, H. G. 1982. Generation of transient cavities in liquids by microsecond ultrasonic pulses of ultrasound. J. Acoust. Soc. Am. 72:1926-1932.

25. Apfel, R. E., and Holland, C. K. 1991. Gauging the likelihood of cavitation from short pulse, low duty cycle diagnostic ultrasound. Ultrasound Med. Biol. 17:179-185.

26. Carr, M. E., and C. L. Hardin. 1987. Fibrin has larger pores when formed in the presence of erythrocytes. Am. J. Physiol. 253:H1069-H1073.

27. Nair, C. H., and D. P. Dhall. 1991. Studies on fibrin network structure: the effect of some plasma proteins. Thromb. Res. 61:315-325.
28. Blinc, A., G. Planinsic, D. Keber, O. Jarh, G. Lahajnar, A. Zidansek, and F. Demsar. 1991. Dependence of blood clot lysis on the mode of transport of urokinase into the clot-A magnetic resonance imaging study in vitro. Thromb. Haemost. 65:549-552.

29. Kandarpa, K., P. A. Drinker, S. J. Singer, and D. Caramore. 1988. Forceful pulsatile local infusion of enzyme accelerates thrombolysis: In vivo evaluation of a new delivery system. Radiology. 168:739-744.

30. Hess, H., H. Ingrisch, A. Mietaschk, and H. Rath. 1982. Local low-dose thrombolytic therapy of peripheral arterial occlusions. N. Engl. J. Med. 307:1627-1630.

31. National Council on Radiation Protection and Measurements. 1992. Exposure criteria for medical diagnostic ultrasound. Part I. Criteria based on thermal mechanisms. NCR Report No. 113. NCRP, Bethesda, MD.

32. National Council on Radiation Protection and Measurements. 1982. Committee No. 66. Biological effects of ultrasound: Mechanisms: Medical Safety. Bethesda, MD.

33. Chater, B. V., and A. R. Williams. 1977. Platelet aggregation induced in vitro by therapeutic ultrasound. Thromb. Haemostas. 38:640-651.

34. Williams, A. R., B. V. Chater, K. A. Allen, M. R. Sherwood, and J. H. Sanderson. 1978. Release of $\beta$-thromboglobulin from human platelets by therapeutic intensities of ultrasound. Br. J. Haematol. 40:133-142.

35. Miller, D. L., W. L. Hyborg, and C. C. Whitcomb. 1979. Platelet aggregation induced by ultrasound under specialized conditions in vitro. Science (Wash. DC). 205:505-507.

36. Frizzell, L. A., D. L. Miller, and W. L. Nyborg. 1986. Ultrasonically induced intravascular streaming and thrombus formation adjacent to a micropipette. Ultrasound Med. Biol. 12:217-222.

37. Walmsley, A. D., W. R. E. Laird, and A. R. Williams. 1987. Intra-vascular thrombosis associated with dental ultrasound. J. Oral Pathol. 16:256-259.

38. Kerr, C. L., D. W. Gregory, K. K. Chan, D. J. Watmough, and D. N. Wheatley. 1989. Ultrasound-induced damage of veins in pig ears, as revealed by scanning electron microscopy. Ultrasound Med. Biol. 15:45-52.

39. Fischell, T. A., M. A. Abbas, G. W. Grant, and R. J. Siegel. 1991. Ultrasonic energy. Effects on vascular function and integrity. Circulation. 84:17831795.

40. Gore, J. M., M. Sloan, T. R. Price, A. M. Y. Randall, E. Bovill, D. Collen, S. Forman, G. L. Knatterud, G. Sopko, M. L. Terrin, and the TIMI Investigators. 1991. Intracerebral hemorrhage, cerebral infarction, and subdural hematoma after acute myocardial infarction and thrombolytic therapy in the thrombolysis in myocardial infarction study. Thrombolysis in myocardial infarction, Phase II, pilot and clinical trial. Circulation. 83:448-459.

41. Stump, D. C., R. M. Califf, E. J. Topol, K. Sigmon, D. Thornton, R. Masek, L. Anderson, D. Collen, and the TAMI Study Group. 1989. Pharmacodynamics of thrombolysis with recombinant tissue-type plasminogen activator Correlation with characteristics of and clinical outcomes in patients with acute myocardial infarction. Circulation. 80:1222-1230. 